\title{
Targeting the Intestinal Barrier to Prevent Gut-Derived Inflammation and Disease: A Role for Intestinal Alkaline Phosphatase
}

\author{
Florian Kühn $^{\text {a }}$ Ruifeng Duan ${ }^{a}$ Matthias $\|_{m e r}{ }^{a}$ Ulrich Wirth ${ }^{a}$ \\ Fatemeh Adiliaghdam $^{b}$ Tobias S. Schiergens ${ }^{a}$ Joachim Andrassy ${ }^{a}$ \\ Alexandr V. Bazhin ${ }^{a}$ Jens Werner ${ }^{a}$ \\ a Department of General, Visceral and Transplant Surgery, University Hospital of LMU Munich, Munich, Germany; \\ ${ }^{b}$ Department of Surgery, Massachusetts General Hospital, Harvard Medical School, Boston, MA, USA
}

\section{Keywords}

Intestinal alkaline phosphatase - Lipopolysaccharides · Gut barrier · Endotoxemia $\cdot$ Microbiome $\cdot$ Diabetes $\cdot$ Metabolic syndrome

\begin{abstract}
Background: Intestinal alkaline phosphatase (IAP) as a tissue-specific isozyme of alkaline phosphatases is predominantly produced by enterocytes in the proximal small intestine. In recent years, an increasing number of pathologies have been identified to be associated with an IAP deficiency, making it very worthwhile to review the various roles, biological functions, and potential therapeutic aspects of IAP. Summary: IAP primarily originates and acts in the intestinal tract but affects other organs through specific biological axes related to its fundamental roles such as promoting gut barrier function, dephosphorylation/detoxification of lipopolysaccharides (LPS), and regulation of gut microbiota. Key Messages: Numerous studies reporting on the different roles and the potential therapeutic value of IAP across species have been published during the last decade. While IAP deficiency is linked to varying degrees of physiological dysfunctions across multiple organ systems, the supplementation of IAP has been proven to be beneficial in several translational and clinical studies. The increasing evidence of the salutary functions of IAP underlines the significance of the naturally occurring brush border enzyme.
\end{abstract}

(C) 2021 S. Karger AG, Basel
F.K. and R.D. share first authorship.

\section{Introduction}

As an isoenzyme of alkaline phosphatase (AP), intestinal AP (IAP) is predominantly produced and secreted by enterocytes in the duodenum, and at lower levels in the jejunum, ileum, and colon [1]. IAP molecules can be found in high concentrations within luminal vesicles secreted by enterocytes on the brush border of the microvilli. Though mostly membrane bound, a small amount of IAP is bidirectionally released into the blood as well as into the lumen [2]. IAP functions to inhibit lipopolysaccharides (LPS) and other inflammatory mediators responsible for endotoxemia and appears to be an important positive regulator of gut barrier function and microbial homeostasis (Fig. 1). Based on the functional roles for IAP, in particular its salutary role in gut barrier integrity and its ability to inhibit bacteria-derived inflammatory mediators, its deficiency has been linked to several pathologies and disorders (Fig. 2). The functions of IAP have been unraveled in several basic, translational, and clinical studies underlining the importance of this gut brush border enzyme. The purpose of this review is to discuss the various roles of IAP within the gut and its connected axes. 


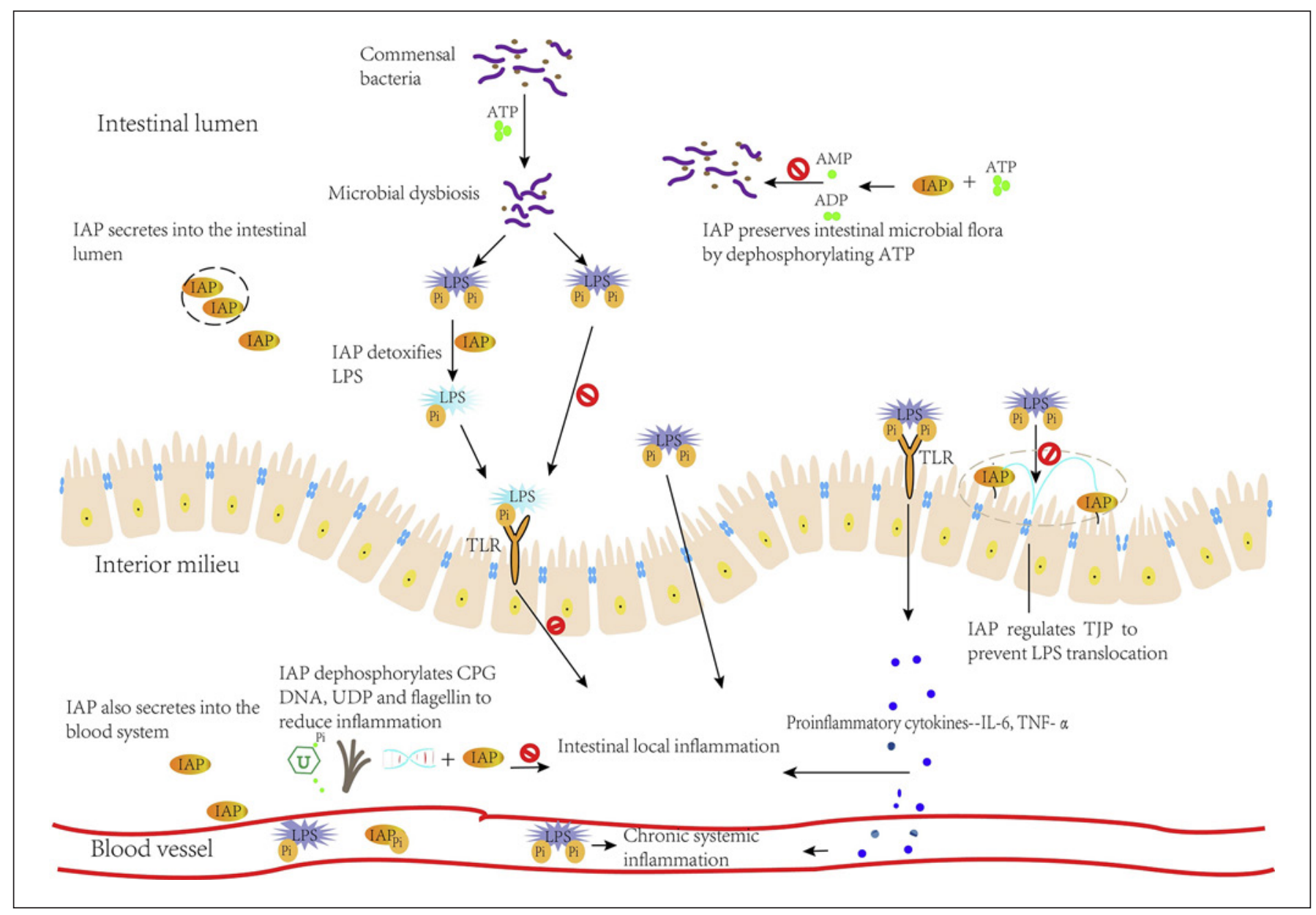

Fig. 1. Working mechanisms of IAP. High levels of ATP lead to intestinal dysbiosis. IAP can dephosphorylate ATP to other nucleotides and thus regulate the number and diversity of commensal bacteria. LPS causes TLR4 activation which further promotes the release of inflammatory mediators. LPS can transfer through the intercellular space or bind to chylomicrons which leads to more LPS absorption in the context of an HFD. Released inflammatory mediators and absorbed LPS can cause local intestinal inflamma- tion and chronic systemic inflammation. IAP removes 1 of the 2 phosphate groups from LPS and reduces its toxicity; dephosphorylated LPS can still bind to TLR4 but mainly acts as a TLR antagonist, thus inhibiting downstream intracellular signaling. IAP also regulates the levels of intestinal intercellular tight junction proteins (TJP) and their cellular localization and reduces the translocation of LPS.

\section{Functions and Working Mechanisms of IAP}

\section{Detoxification of Bacterial Byproducts}

IAP exhibits anti-inflammatory properties based on its ability to dephosphorylate LPS, flagellin, cytosinephosphate-guanosine (CpG) DNA, and uridine diphosphate (UDP) [3]. LPS activates toll-like receptor 4 (TLR4) and promotes translocation of nuclear factor $(\mathrm{NF})-\kappa \mathrm{B}$ to the nucleus. This leads to the release of inflammatory mediators including interleukin (IL)-1, IL-6, and tumor necrosis factor (TNF)- $\alpha[4,5]$. IAP removes one of the 2 phosphate groups from the lipid-A moiety of LPS and reduces its toxicity by a 100-fold [6]. Dephosphorylated LPS can still bind to TLR4 but mainly acts as a TLR4 antagonist, thus inhibiting downstream intracellular signaling [6]. Flagellin and CpG DNA can induce IL-8 se- cretion in HT29 cells or THP-1 cells in a dose-dependent manner [7]. Both of them can be dephosphorylated by IAP [7]. Pretreatment of flagellin and CpG DNA with IAP caused a $>40 \%$ reduction in IL- 8 secretion by target cells, which was also IAP dose dependent [7]. It is well known that inflammation can stimulate host cells to release nucleotides, including UDP, which further results in the release of inflammatory cytokines [8]. IAP can efficiently dephosphorylate UDP in a dose-dependent manner [8].

\section{Promoting Gut Barrier Function and Regulation of Gut Microbiota}

IAP promotes gut barrier function by regulating the levels of key tight junction proteins and their cellular localization $[3,9]$. Although the precise molecular mecha- 


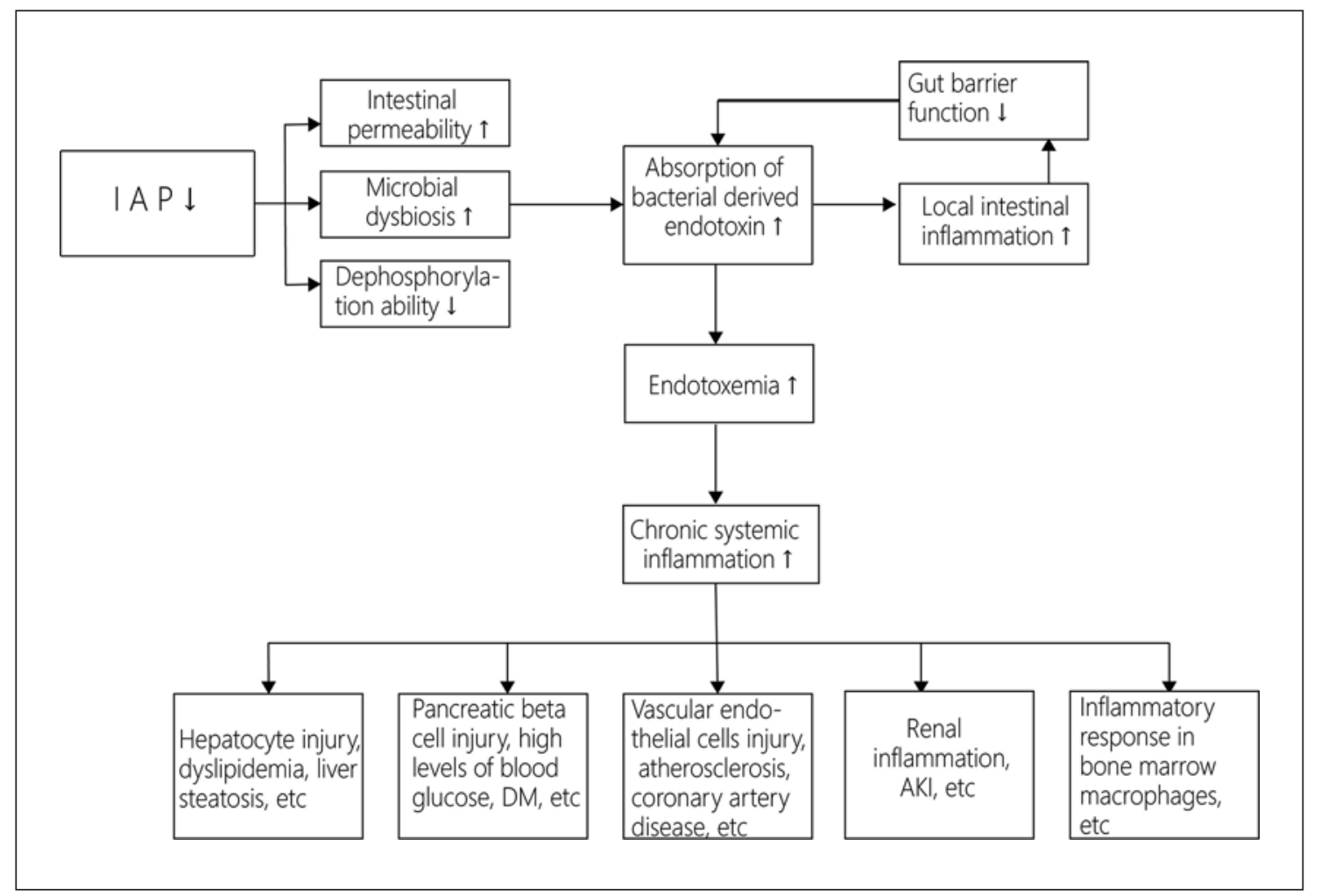

Fig. 2. IAP deficiency results in various pathologies/disorders caused by endotoxemia and chronic systemic inflammation. $\downarrow$ indicates a decrease, and $\uparrow$ indicates an increase, in the parameters. DM, diabetes mellitus.

nisms of how IAP affects tight junction proteins are still unknown, it has been demonstrated that IAP knockout (KO) mice have lower zonula occludens (ZO)-1, ZO-2, and occludin levels, while overexpression of the IAP gene results in a significant increase in ZO- 1 and ZO-2 mRNA levels in Caco-2 and T84 cells [3, 9]. Besides, exogenous IAP promoted the expression of claudin $1, \mathrm{ZO}-1$, and $\mathrm{ZO}-3$ in fed mice and significantly increased the levels of claudin1, occludin, ZO-1, ZO-2, and ZO-3 in starved mice $[3,9]$. Loss of IAP is associated with a significant reduction in expression levels of tight junction proteins in intestinal tissues [10]. Furthermore, IAP has been shown to preserve intestinal homeostasis of gut microbiota in mice $[10,11]$. Previous studies have demonstrated that a high levels of adenosine triphosphate (ATP) lead to a disturbance of intestinal microbial homeostasis. Thus, the influence of IAP on regulation of the number and diversity of commensal bacteria in the intestine may be achieved by dephosphorylation of ATP [12].

\section{Regulation of Bicarbonate Secretion and Intestinal Surface $p H$}

Bicarbonate secretion by enterocytes protects the duodenal mucosa against gastric acids and pepsin by creating a higher $\mathrm{pH}$ on the apical surface of enterocytes [13]. Here, IAP forms a regulatory loop together with extracel- lular detrimental ATP, and G-protein coupled purinergic receptor P2Y1 (located on the brush border membrane of duodenal villi), to control intestinal surface $\mathrm{pH}$ [13]; ATP stimulates P2Y receptors to secrete bicarbonate ions. Bicarbonate neutralizes the acidic environment and promotes IAP activity [12]. Conversely, a high duodenal surface $\mathrm{pH}$ can augment IAP activity, thereby increasing the rate of ATP degradation, decreasing the bicarbonate release and forming a negative feedback loop [12].

\section{Regulation of $\mathrm{Ca}, \mathrm{Pi}$, and Lipid Absorption}

IAP is considered to play a significant role in regulation of lipid absorption in the small intestine through CD36. It is generally accepted that CD36 facilitates the uptake and transportation of lipids in the small intestine [14]. Phosphorylation of CD36 inhibits long-chain fatty acid uptake. IAP functions to dephosphorylate CD36 (pCD36) and leads to an enhanced uptake of long-chain fatty acids [14]. Thereby, pCD36 represents a physiological substrate for IAP as the interaction between pCD36 and IAP optimizes long-chain fatty-acid transport.

The transient receptor potential vanilloid type 5 and 6 (TRPV5/6) is related to intestinal transcellular $\mathrm{Ca}^{2+}$ transportation, and it is known that its activity is regulated by luminal $\mathrm{pH}$ [15]. IAP appears to affect TRPV5/6 channel 
Table 1. Roles for IAP in different biological axes and within the gut

\begin{tabular}{lll}
\hline & $\begin{array}{l}\text { IAP deficiency indicates and/ } \\
\text { or causes these dysfunctions }\end{array}$ & $\begin{array}{l}\text { IAP supplementation prevents and/or attenuate } \\
\text { these dysfunctions }\end{array}$ \\
\hline Gut & NEC [31] and IBD [13] & $\begin{array}{l}\text { Salmonella and C. difficile infection [24, 26], } \\
\text { NEC [29, 30], and UC [32] }\end{array}$ \\
\hline Gut-liver axis & Aging-related liver change [10] and cirrhosis [34] & ALD [36] and cirrhosis [34] \\
\hline Gut-pancreas axis & T2DM [48] & Metabolic syndrome [10] \\
\hline Gut-heart axis & IHD [50] & $\begin{array}{l}\text { AMI-induced inflammation [55], } \\
\text { myocardial dysfunction [53], and } \\
\text { complications of AMI [53] }\end{array}$ \\
\hline Gut-kidney axis & & $\begin{array}{l}\text { Sepsis-induced AKI [61], renal inflammation [66], } \\
\text { and damage of I/R-induced AKI [66] }\end{array}$ \\
\hline Gut-bone axis & $\begin{array}{l}\text { IAP deficiency increases cortical thickness, the volume } \\
\text { of intracortical bone, and trabecular connectivity [70] }\end{array}$ & \\
\hline \multicolumn{2}{c}{ AMI, myocardial infarction; ALD, alcoholic liver disease; I/R, ischemia-reperfusion. } \\
\hline
\end{tabular}

activity through luminal $\mathrm{pH}$ changes, thereby controlling $\mathrm{Ca}^{2+}$ absorption. In rats, type II sodium-dependent inorganic phosphate $(\mathrm{Pi})$ transporter $(\mathrm{Npt} 2 \mathrm{~b})$ mediates intestinal transcellular Pi transport on the apical side of epithelial cells [16], IAP gene deletion suppresses $\mathrm{Na}^{+}$-dependent $\mathrm{Pi}$ transport by reducing intestinal $\mathrm{Npt} 2 \mathrm{~b}$ protein levels in mice [16].

So far, most of the functions and working mechanisms of IAP have been identified either in vitro or in animal models. Even though more translational data is needed to better judge the actual role of IAP in humans, the multiple functions of IAP have been linked to important physiological mechanisms across multiple organ systems. IAP has already been tested in a number of clinical studies, and its efficacy and safety have been proven, for example, in blocking endotoxemia in septic patients and in preventing flares in patients with ulcerative colitis (UC) [17, 18]. As a naturally occurring enzyme, IAP can be administered orally, intravenously, and as rectal suppository [19]. However, there are some distinctions among these routes. Some studies chose systemic IAP delivery, which allowed for more efficient absorption and precise dosing $[20,21]$. In animal models, oral IAP supplementation led to significantly higher stool IAP activity levels, which indicated that most of this enzyme could remain stable in the intestine after oral ingestion [10]. Moreover, oral supplementation of IAP could not only reduce the systemic inflammation but also stimulate the endogenous IAP, whereas intravenous administration was only able to decrease systemic inflammation $[13,22]$. Intrarectal administration of IAP was the best choice for reducing colitis in rats, because direct IAP administration in the rectum probably avoided partial IAP depletion during its transit through the stomach [23].

\section{Biological Axes of IAP}

We herein review the various roles of IAP and its different biological axes (Table 1) and discuss potential therapeutic targets.

\section{The Role of IAP within the Gut}

The gut barrier is a highly specialized entity that coordinates nutrient absorption and protects organisms from potentially harmful luminal factors. Commensal bacteria residing in the gastrointestinal (GI) tract play a pivotal role in the development of our immune system and its physiological functions [24]. On the downside, dysbiosis may favor intestinal barrier disruption, further promotes the growth of pathogenic bacteria, and is associated with an increased susceptibility to certain diseases [25]. As the first physical barrier, the intestinal epithelial cell layer has an important role in protecting organisms from the luminal contents. The tight junction proteins that seal and control the paracellular space are crucial for maintaining the integrity of the epithelial barrier. Abnormal expression of these proteins could compromise intestinal integrity and induce a "leaky gut," thereby resulting in various intestinal and systemic disorders [26]. Since IAP is able to regulate gut microbiota, promote gut barrier integrity, and dephosphorylate bacterial derived endotoxins, it plays a crucial role in preserving intestinal homeostasis $[11,13]$.

\section{IAP and Salmonella spp.}

IAP deficiency has been associated with intestinal inflammation in several animal models as well as in human studies $[27,28]$. Salmonella bacteria are common and 
widespread enteric pathogens. Recurrent low-dose and nonlethal Salmonella infection induce a form of longlasting IAP deficiency that might be contributing to the mechanism of inflammation and tissue damage caused by Salmonella spp. [29]. Salmonella infection induces endogenous neuraminidase (Neu) activity among enterocytes of the duodenum in a TLR4-dependent manner. This elevation of Neu activity could impair normal IAP molecule conformation, thus reducing its activity in the colon and triggering intestinal inflammation. IAP supplementation and inhibition of Neu activity are similarly effective at mitigating the effects of repeated Salmonella spp. infection [29].

\section{IAP Exerts Protective Effects against Chronic Colitis}

Ramasamy et al. [30] showed that endogenous IAP plays a pivotal role in protecting the host against dextran sulfate sodium-induced colitis in mice. In addition, exogenous oral supplementation of IAP significantly attenuated inflammation in both wild-type (WT) and IAP-KO groups, indicating the potential therapeutic role for this enzyme. In another model, IAP treatment could also decrease tissue myeloperoxidase activity and proinflammatory cytokine levels compared to control groups in irradiated Wiskott-Aldrich syndrome protein (WASp)-deficient mice with chronic spontaneous colitis [30]. IAP may exert protective effects against chronic colitis, either through detoxification of microbial endotoxins such as LPS and flagellins and/or by suppression of proinflammatory cytokine release [30].

\section{IAP Prevents Antibiotic-Associated Infections}

A study by Alam et al. [31] showed that overuse of antibiotics leads to a disturbance of the number and composition of intestinal microbiota, increasing the host susceptibility to enteric pathogens. Oral supplementation with IAP during antibiotic treatment protected the mice from infections with Salmonella enterica and Clostridium difficile; exogenous IAP also decreased colonic inflammation, maintained their weight, and improved survival in mice [31]. The ability of this enzyme to rapidly restore the commensal gut microflora in the context of antibiotic treatment may contribute to the beneficial effects of IAP seen in this study. Oral administration of IAP during antibiotic treatment may represent a novel way to prevent bacterial infections caused by Salmonella spp., C. difficile, and perhaps other enteric pathogens in humans [31].

\section{IAP for Diagnosis and Treatment of Necrotizing \\ Enterocolitis}

Necrotizing enterocolitis (NEC) is a serious intestinal inflammatory disease of the neonate associated with an increased gut permeability to macromolecules [32]. In that context, preterm rat pups showed decreased IAP ac- tivity compared to full-term rat pups [33]. IAP was shown to significantly decrease intestinal and systemic inflammation and intestinal damage and maintain gut barrier function in rat pups exposed to stressors inducing the development of NEC $[34,35]$. Thus, daily enteral IAP supplementation beginning on day 0 could be a promising treatment for prevention of the development of NEC. Administration of IAP after the onset of NEC did not decrease intestinal inflammation or structural damage in rat pups, which suggests early IAP supplementation for the high-risk group of NEC [33]. In a diagnostic study, high amounts of IAP protein in stool and low IAP enzyme activity were related to the diagnosis of NEC in premature infants. Importantly, IAP was associated with NEC and not associated with sepsis or other non-GI infections. This finding indicates that IAP might also serve as a useful biomarker for NEC and lead to lower morbidity and mortality in patients with NEC [36].

\section{Patients with Inflammatory Bowel Disease Have} Lower IAP Activities and IAP Supplementation Attenuates Inflammatory Bowel Disease

The 2 major forms of inflammatory bowel disease (IBD), i.e., Crohn's disease (CD) and UC, are characterized by chronic inflammation, increased gut permeability, and intestinal dysbiosis. IBD patients display a decrease in the expression levels and activity of endogenous IAP [37]. A recent study indicates that, in patients with $\mathrm{CD}$ and UC, IAP mRNA expression levels are much lower in inflamed colonic tissues compared to noninflamed tissues. Moreover, also the noninflamed tissues of IBD patients had lower IAP mRNA expression levels than control samples from healthy subjects. Noninflamed and inflamed colonic tissues from IBD patients both had no LPS-dephosphorylating capacity in vitro [13].

Increased intestinal permeability, dysbiosis, and a higher TLR4 expression in intestinal epithelium may have a crucial role in IBD pathogenesis [37]. These disorders seem to be attributed to the lower intestinal synthesis and activity of endogenous IAP, but the pathomechanism of IBD concerning IAP deficiency has not been fully explored. Even so, in a clinical trial, IAP supplementation daily over a 7-day course in patients with UC was associated with short-term improvement in disease activity scores, clinical effects, and a reduction of C-reactive protein and stool calprotectin [37].

Interestingly, IAP activity from inflamed mucosa was higher than that from noninflamed mucosa in CD patients without clinical recurrence, whereas in patients with clinical recurrence, there was no significant difference in IAP between noninflamed and inflamed mucosa. A lower expression of endogenous IAP in inflamed tissue may, hence, predict worse prognosis in patients with $C D$ [38]. 
Taken together, IAP appears to play an important role in maintaining gut homeostasis by preventing dysbiosis and increased gut permeability.

\section{Gut-Liver Axis}

The intestinal barrier is located at the interface between the host and a microbial population that is the source of important mediators of intestinal and hepatic inflammation [39]. In addition to nutrients, portal-venous blood carries factors derived from intestinal microbiota and immune response products to the liver [40]. Hence, the liver is the primary recipient of gut-derived products, making it susceptible to the effects of gut-derived toxins. Several studies have indicated that IAP targets the gut-liver axis to prevent liver damage from potentially harmful luminal factors. In this context, Kühn et al. [10] found that a lack of IAP was associated with more severe aging-related liver inflammation, steatosis, and increased proinflammatory mediators (cytokines and LPS) of portal serum in mice. In this study, age-related changes in the liver (inflammation, hepatocyte vacuolation, scattered neutrophils, etc.) increased with age. Furthermore, these changes were significantly more obvious in the liver of IAP-KO mice than in their WT counterparts. Moreover, the macrosteatosis score was significantly higher in IAP-KO animals. The levels of LPS in systemic and portal sera were significantly increased in IAP-deficient mice. In vitro, portal serum from IAP-KO mice caused a much more pronounced inflammatory response in target cells than serum from WT mice [10].

Ethanol administration has been proven to be associated with decreased IAP levels in mice [41]. When studying the effect of IAP on alcohol-induced hepatosteatosis in mice, oral supplementation with IAP resulted in significantly lower serum alanine aminotransferase (ALT) levels in comparison with the ethanol-alone group in the acute binge model. IAP administration delayed the development of alcohol-induced fatty liver and prevented alcohol-induced endotoxemia and liver inflammation [41]. Interestingly, IAP also delayed alcohol-induced stellate cell activation and the lipogenic effect, both of which played major roles in the development of liver steatosis [41].

Patients with alcoholic liver disease exhibit significantly higher levels of LPS due to acute and chronic alcohol intake [42, 43]. LPS appears to play a major role in alcoholic liver inflammation and the pathogenesis of alcoholic liver disease by binding to the TLR 4 complex [ 42 , 44]. Thereby, it activates an innate immune response in the liver followed by increased production of inflammatory mediators, especially TNF- $\alpha$, IL- $1 \beta$, and reactive oxygen species (ROS), contributing to development of fatty liver, inflammation, and fibrosis [45, 46]. Furthermore, LPS aggravates alcohol-induced hepatic microvascular dysfunction, liver injury, and apoptosis [47-49]. IAP supplementation has been shown to compensate for junctional protein losses and gut barrier dysfunction caused by alcohol intake in mice, and detoxify bacterial-derived inflammatory factors, thus reducing their translocation into the portal vein [41].

A study by Liu et al. [39] demonstrated that human IAP activity is reduced in patients with liver cirrhosis and negatively correlates with the Child-Pugh liver classification. Murine stool IAP activity as well as expression levels also declined during liver fibrosis induced by common bile duct ligation (CBDL) or carbon tetrachloride-4 (CCl4) injection. In these mice, intestinal tight junction protein expression and gut barrier function were impaired and IAP-KO mice showed a significantly worse gut barrier function which further aggravated fibrosis [39]. Oral IAP supplementation increased tight junction protein gene expression and improved intestinal barrier function in both animal models of liver fibrosis. Although IAP rescued liver fibrosis in both WT and IAP-KO mice in both CBDL and CCl4 models, it did not attenuate fibrosis in TLR4-KO mice indicating that IAP prevents liver fibrosis through the TLR4 pathway [39].

Hence, IAP-deficiency makes the liver more susceptible to harmful factors from the gut, whereas IAP supplementation prevents those factors from entering the portal-venous system by luminal detoxification and promoting gut barrier function.

\section{Gut-Pancreas Axis}

Recently, several studies have investigated the role of gut microbiota and gut barrier function in pancreatic disease $[50,51]$. The role of IAP in diabetes and metabolic syndrome has been examined in human and translational studies $[52,53]$. A case-control study in metabolic disease indicated that type 2 diabetes mellitus (T2DM) patients have approximately $50 \%$ less fecal IAP compared to healthy controls. High IAP can protect against diabetes irrespectively of obesity, whereas healthy controls with low IAP levels also appeared to be vulnerable to the development of metabolic syndrome [53].

Kaliannan et al. [52] revealed that IAP-KO mice suffered from hyperinsulinemia and glucose intolerance. Furthermore, in that study, IAP-KO mice put on a highfat diet (HFD) exhibited T2DM symptoms such as higher fasting glucose levels and an abnormal glucose tolerance. An HFD is known to lead to a higher proportion of LPScontaining microbiota [54], and that LPS binds to fatty acids which lead to more LPS absorption in the context of HFD [55]. In return, absorbed LPS can cause local intes- 
tinal inflammation and increase intestinal permeability, resulting in increased translocation [54]. The endotoxemia caused by gut-derived endotoxins induces a chronic systemic inflammation that can lead to hepatocyte and pancreatic $\beta$-cell injury, dyslipidemia, high levels of blood glucose, and even T2DM [54]. Due to the ability of IAP to improve gut barrier function, regulate intestinal microbiota, and dephosphorylate various proinflammatory factors, mice receiving an HFD and IAP supplementation exhibited a better glucose tolerance, less insulin resistance, and postprandial hyperinsulinemia compared to mice receiving the HFD alone [52]. Both orally supplemented and endogenous IAP inhibited the absorption of LPS occurring with dietary fat. Thus, oral IAP supplementation can not only prevent but even reverse HFDinduced metabolic syndrome in mice [52]. Furthermore, IAP was shown to be associated with an improved lipid profile and lower blood glucose, urea nitrogen, and serum liver enzyme levels [10]. Coadministration of IAP with antibiotics can prevent metabolic syndrome via maintenance of intestinal microbial homeostasis in mice [56].

IAP is likely to have similar effects on preventing and delaying the development of T1DM. High-risk diabetesprone rats showed alterations in the tight-junction protein claudin and a higher intestinal permeability compared to low-risk diabetes-resistant rats [57]. Li and Atkinson [58] showed that a leaky gut and microbiota imbalance caused an autoimmune cascade leading to $\beta$-cell autoimmunity and insulitis and eventually accelerating the development of T1DM. Based on these results, it was supposed that IAP may at least partly be involved in the pathogenesis of T1DM.

Given the facts above, IAP might also serve as a biochemical marker for identifying the incipient metabolic syndrome which is a critical step to controling pancreatic metabolic disease. Supplementing exogenous IAP or increasing endogenous IAP production are new potential therapeutic approaches for preventing and treating pancreatic metabolic disorders including diabetes.

\section{Gut-Heart Axis}

Recent studies have found an obvious correlation between IAP deficiency and ischemic heart disease (IHD) $[55,59,60]$. Here, IHD patients had approximately $30 \%$ lower levels of IAP in stool samples in comparison to the healthy controls. The generalized linear modeling verified a strong association of IAP with IHD. Multiple logistic regression analysis controlling for physical and biochemical characteristics showed an independent inverse relationship between the IHD status and levels of IAP [55]. The authors discuss the pathogenesis of IHD caused by IAP deficiency as follows: IAP deficiency increases in-

IAP Targets Gut-Derived Inflammation and Disease testinal permeability and intraluminal bacteria-derived LPS concentration $[52,61]$, both of which contribute to excessive translocation of LPS into the systemic circulation, causing varying degrees of endotoxemia. Subsequently, endotoxemia leads to chronic systemic inflammation, which has already been proven to cause damage to vascular endothelial cells followed by atherosclerosis and coronary artery disease [52].

Due to its detoxification ability, IAP also has beneficial effects on the prognosis of IHD. In mice models of acute myocardial infarction, intravenous administration of bovine IAP (bIAP) resulted in a significant decrease of the pro-inflammatory cytokines IL- 6 and IL- $1 \beta$ and an obvious reduction of mast cell activation in the acute phase after acute myocardial infarction compared to controls [60]. Therefore, IAP might decrease myocardial dysfunction and the incidence of cardiogenic shock complications after acute myocardial infarction [59]. A clinical study reported that intravenous bolus administration and continuous infusion of IAP in patients undergoing coronary artery bypass grafting induced an increased release of liver-type tissue nonspecific AP (TNAP) into the systematic circulation. The increased level of TNAP in blood could be the body's way of strengthening its innate immune defense against a massive ischemic insult [62]. The amount of additional endogenous TNAP released into the circulation was in proportion to the total concentration of bolus IAP being supplied [63].

Although the precise mechanism is not fully understood, IAP deficiency seems to play a certain role in the pathogenesis of IHD. Exogenous IAP supplementation might be a novel therapeutic approach for the prevention of IHD.

\section{Gut-Kidney Axis}

Dysbiosis of the intestinal microbiota has been reported in various renal associated diseases such as systemic lupus erythematosus or chronic kidney disease [64]. The accumulation of uremic toxins may lead to mucosal irritation and structural damage [64]. In turn, GI disorders may play a significant role in increasing the risk of kidney disease and its progression, since disruption of the GI wall and microbial dysbiosis increase the translocation of toxins associated with nephropathy, which range from smaller molecules such as p-cresyl sulfate to high molecular inflammatory products such as LPS [65]. Elevated systemic levels of toxins are associated with the development and progression of renal diseases [64]. IAP is likely to play an important role along the gut-kidney axis by promoting mucosal defense and suppressing inflammatory mediators.

Sepsis is a systemic inflammatory syndrome leading to serious tissue and organ damage. Acute kidney injury 
(AKI) often occurs secondary to sepsis and leads to chronic kidney failure. IAP has been demonstrated to have beneficial effects on patients with sepsis and AKI in clinical trials. These trials have demonstrated that IAP exhibits protective effects by dephosphorylation of LPS [66]. According to 2 other clinical studies, IAP treatment increased endogenous creatinine clearance and reduced the urinary excretion of renal injury markers during sepsis-related AKI. Furthermore, IAP reduced the duration of and the need for renal replacement therapy $[18,20]$.

Human recombinant chimeric intestinal AP (RecAP) possesses the dephosphorylating functionality of IAP [21]. Therefore, it can reduce the level of proinflammatory cytokines by dephosphorylating LPS as well as ATP and adenosine diphosphate, released by proximal tubule cells during inflammation in vitro [67]. Through this mechanism, RecAP could ameliorate renal inflammation in a rat model and human proximal tubule epithelial cells in vitro $[67,68]$. In vivo, RecAP prevented ischemia-reperfusion-induced renal inflammation and alterations of renal hemodynamics after reperfusion in rodent models of ischemia-reperfusion-induced AKI. Moreover, RecAP also ameliorated renal inflammation and damage during LPS-induced shock in rats [69].

Several studies have shown a marked reduction of intestinal tight junction proteins in chronic kidney disease. Moreover, intestinal paracellular permeability and gut microbiota play an important role in the development and prognosis of chronic kidney disease [65]. IAP could compensate for the impaired intestinal barrier function and maintain gut homeostasis, thus promoting renal function recovery.

Based on its salutary functions, recombinant IAP is in late clinical trials for sepsis-associated AKI, suggesting that it may soon become available as a therapeutic.

\section{Gut-Bone Axis}

The key bone mineral calcium has been the only known connection between the gut and the bone for a long time. Recent findings indicate that the microbiome and gutderived mediators also affect bone metabolism via the socalled "gut-bone axis" [70].

Next to preserving the homeostasis of intestinal microbiota [11], IAP plays an important role in the regulation of calcium absorption [71]. In a previous study, although no statistical difference between groups was found, a higher percentage of $\mathrm{Ca}^{2+}$ absorption was observed in IAP-deficient mice compared to WT mice. The results also demonstrated better trabecular connectivity of the bone in IAP-KO mice. In the femoral neck fracture test, a significantly higher fracture load was observed in
KO mice [72]. Kuehn et al. [73] found that there was no apparent difference between IAP-deficient and WT mice in the levels of serum $\mathrm{Ca}^{2+}$ or Pi. In this study, older IAP$\mathrm{KO}$ mice showed an isolated increase in serum AP levels, which reflected a state of active bone formation. Serum from IAP-KO mice induced a significantly higher inflammatory response in primary bone marrow macrophages than serum from WT mice [73]. Compared to their WT counterparts, older female IAP-deficient mice showed a dramatic increase in cortical thickness and disruption of the cortex with vasculature and islands of stromal cells on bone histology. Furthermore, older IAP-KO mice displayed a greater volume of intracortical bone with a much more disorganized and chaotic osseous structure on computed tomography (CT) [73]. These IAP-KO bone phenotypes on histology and CT were absent in younger mice, indicating that a lack of IAP seemed to manifest itself in a chronic process [73]. Interestingly, the IAP-KO group also demonstrated a significant decrease in fecal Lactobacillus spp., which could result in a higher bone mineralization and greater bone strength [70]. In short, the loss of IAP leads to chronic changes in the bone, most likely due to dysbiosis and systemic dissemination of proinflammatory mediators.

\section{Additional Clinical Implications for IAP}

Next to the above findings, IAP may have additional clinical implications. Increased gut permeability and chronic low-grade inflammation linked to persistent gut-derived endotoxemia play crucial roles in a variety of age-related diseases. Therefore, Kühn et al. [10] investigated the potential role of IAP in the context of aging and frailty. Here, an age-related increase in gut permeability and gut-derived portal-venous and systemic inflammation were significantly more pronounced in IAP-deficient animals [10]. Furthermore, oral IAP supplementation significantly decreased age-related gut permeability and gut-derived systemic inflammation resulted in less frailty and extended lifespan. The authors concluded that IAP supplementation may represent an effective therapy to counteract the chronic inflammatory state leading to frailty and age-related diseases in humans [10, 74]. Very recently, Adiliaghdam et al. [75] demonstrated that IAP plays a critical role in preservation of the gut barrier function in the setting of burn and wound infection. In that study oral administration of IAP after a burn injury attenuated gut barrier damage, reduced local and systemic inflammation, and improved survival after burn wound infection in mice. These results indicate that IAP might represent a novel approach to treating patients after burn injuries [75]. In addition, peritoneal irrigation with IAP reduced the increased 
permeability of the small intestine induced by the cecal ligation and puncture procedure in mice by $50 \%$. More than that, IAP also significantly enhanced survival in mice undergoing cecal ligation and puncture, likely through a reduction of local inflammation and remote organ damage $[76,77]$. These findings suggest that IAP supplementation may promote the recovery of patients after abdominal surgery.

\section{Future Research Perspectives}

IAP has exerted its various functions in basic and translational studies. The existing studies on IAP encourage the replacement of IAP to support GI health and reduce several gut-derived acute and chronic inflammatory diseases. All of the studies described to date support the safety of both parenteral and oral administration of this important enzyme. Pharmaceutical companies are currently working on an improved version of recombinant IAP which will probably be available in the foreseeable future. With the emergence of more clinical studies on IAP, we believe that IAP treatment will play an important role in various GI disorders, especially for inflammatory pathologies caused by gut barrier dysfunction or endotoxemia. IAP could be applied either as an add on therapy next to antibiotics or as a targeted therapy in septic patients to detoxify LPS and improve gut barrier integrity. In addition, IAP could be administered in patients undergoing surgery in order to preserve intestinal homeostasis and prevent endotoxemia.

\section{Conclusion}

The gut has become increasingly recognized as the source of multiple pathologies throughout the human body. IAP as a naturally occurring, multifunctional gut brush border enzyme impacts various other organs via specific biological axes. While IAP deficiency is linked to varying degrees of physiological dysfunctions across multiple organ systems, supplementation of IAP has been proven to be beneficial in several translational and clinical studies. The increasing evidence of the salutary functions of IAP underlines the significance of the naturally occurring brush border enzyme.

\section{Conflict of Interest Statement}

The authors have no disclosures or conflicts of interests to report.

\section{Funding Sources}

F.K. was supported by a research grant from the FriedrichBaur-Stiftung (RG No. 28/19).

\section{Author Contributions}

R.D., F.K., and A.V.B. gathered and performed the literature review and wrote this paper. F.A., M.I., T.S.S., U.W., J.A., and J.W. revised this paper for important intellectual content. All of the authors approved the final version of this work for publication.

\section{References}

1 Domar U, Nilsson B, Baranov V, Gerdes U, Stigbrand T. Expression of intestinal alkaline phosphatase in human organs. Histochemistry. 1992 Dec;98(6):359-64.

2 Estaki M, DeCoffe D, Gibson DL. Interplay between intestinal alkaline phosphatase, diet, gut microbes and immunity. World J Gastroenterol. 2014 Nov;20(42):15650-6.

3 Hamarneh SR, Mohamed MM, Economopoulos KP, Morrison SA, Phupitakphol T, Tantillo TJ, et al. A novel approach to maintain gut mucosal integrity using an oral enzyme supplement. Ann Surg. 2014 Oct; 260(4):706-14.

4 Cario E. Bacterial interactions with cells of the intestinal mucosa: toll-like receptors and NOD2. Gut. 2005 Aug;54(8):1182-93.

5 Janssens S, Beyaert R. Role of Toll-like receptors in pathogen recognition. Clin Microbiol Rev. 2003 Oct;16(4):637-46.

6 Ghosh SS, Wang J, Yannie PJ, Ghosh S. Intestinal Barrier Dysfunction, LPS Translocation, and Disease Development. J Endocr Soc. 2020 Feb; 4(2): bvz039.

7 Chen KT, Malo MS, Moss AK, Zeller S, Johnson $\mathrm{P}$, Ebrahimi F, et al. Identification of spe- cific targets for the gut mucosal defense factor intestinal alkaline phosphatase. Am J Physiol Gastrointest Liver Physiol. 2010 Aug; 299(2):G467-75.

8 Moss AK, Hamarneh SR, Mohamed MM, Ramasamy S, Yammine H, Patel P, et al. Intestinal alkaline phosphatase inhibits the proinflammatory nucleotide uridine diphosphate. Am J Physiol Gastrointest Liver Physiol. 2013 Mar;304(6):G597-604.

9 Liu W, Hu D, Huo H, Zhang W, Adiliaghdam F, Morrison S, et al. Intestinal Alkaline Phosphatase Regulates Tight Junction Protein Levels. J Am Coll Surg. 2016 Jun;222(6):1009-17.

10 Kühn F, Adiliaghdam F, Cavallaro PM, Hamarneh SR, Tsurumi A, Hoda RS, et al. Intestinal alkaline phosphatase targets the gut barrier to prevent aging. JCI Insight. 2020 Mar;5(6):134049.

11 Malo MS, Alam SN, Mostafa G, Zeller SJ, Johnson PV, Mohammad N, et al. Intestinal alkaline phosphatase preserves the normal homeostasis of gut microbiota. Gut. 2010 Nov;59(11):1476-84.

12 Zaher DM, El-Gamal MI, Omar HA, Aljareh SN, Al-Shamma SA, Ali AJ, et al. Recent ad- vances with alkaline phosphatase isoenzymes and their inhibitors. Arch Pharm (Weinheim). 2020 May;353(5):e2000011.

13 Lallès JP. Intestinal alkaline phosphatase: multiple biological roles in maintenance of intestinal homeostasis and modulation by diet. Nutr Rev. 2010 Jun;68(6):323-32.

14 Buchet R, Millán JL, Magne D. Multisystemic functions of alkaline phosphatases. Methods Mol Biol. 2013;1053:27-51.

15 Brun LR, Brance ML, Lombarte M, Lupo M Di Loreto VE, Rigalli A. Regulation of intestinal calcium absorption by luminal calcium content: role of intestinal alkaline phosphatase. Mol Nutr Food Res. 2014 Jul;58(7): 1546-51.

16 Sasaki S, Segawa H, Hanazaki A, Kirino R, Fujii T, Ikuta K, et al. A Role of Intestinal Alkaline Phosphatase 3 (Akp3) in Inorganic Phosphate Homeostasis. Kidney Blood Press Res. 2018;43(5):1409-24.

17 Lukas M, Drastich P, Konecny M, Gionchetti P, Urban O, Cantoni F, et al. Exogenous alkaline phosphatase for the treatment of patients with moderate to severe ulcerative colitis. Inflamm Bowel Dis. 2010 Jul;16(7):1180-6. 
18 Pickkers P, Heemskerk S, Schouten J, Laterre PF, Vincent JL, Beishuizen A, et al. Alkaline phosphatase for treatment of sepsis-induced acute kidney injury: a prospective randomized double-blind placebo-controlled trial. Crit Care. 2012 Jan;16(1):R14.

19 Lallès JP. Recent advances in intestinal alkaline phosphatase, inflammation, and nutrition. Nutr Rev. 2019 Oct;77(10):710-24.

20 Heemskerk S, Masereeuw R, Moesker O, Bouw M P, van der Hoeven J G, Peters W H, et al. Alkaline phosphatase treatment improves renal function in severe sepsis or septic shock patients. Crit Care Med. 2009 Feb; 37(2): 417-23, e1.

21 Peters E, Stevens J, Arend J, Guan Z, Raaben $\mathrm{W}$, Laverman $\mathrm{P}$, et al. Biodistribution and translational pharmacokinetic modeling of a human recombinant alkaline phosphatase. Int J Pharm. 2015 Nov;495(1):122-31.

22 Lallès JP. Intestinal alkaline phosphatase: novel functions and protective effects. Nutr Rev. 2014 Feb;72(2):82-94.

23 Martínez-Moya P, Ortega-González M, González R, Anzola A, Ocón B, HernándezChirlaque C, et al. Exogenous alkaline phosphatase treatment complements endogenous enzyme protection in colonic inflammation and reduces bacterial translocation in rats. Pharmacol Res. 2012 Aug;66(2):144-53.

24 Koch S, Nusrat A. The life and death of epithelia during inflammation: lessons learned from the gut. Annu Rev Pathol. 2012;7(1):3560.

25 Takiishi T, Fenero CI, Câmara NO. Intestinal barrier and gut microbiota: shaping our immune responses throughout life. Tissue Barriers. 2017 Oct;5(4):e1373208.

26 Chelakkot C, Ghim J, Ryu SH. Mechanisms regulating intestinal barrier integrity and its pathological implications. Exp Mol Med. 2018 Aug;50(8):1-9.

27 Bates JM, Akerlund J, Mittge E, Guillemin K. Intestinal alkaline phosphatase detoxifies lipopolysaccharide and prevents inflammation in zebrafish in response to the gut microbiota. Cell Host Microbe. 2007 Dec;2(6):371-82.

28 Tuin A, Poelstra K, de Jager-Krikken A, Bok L, Raaben W, Velders MP, et al. Role of alkaline phosphatase in colitis in man and rats. Gut. 2009 Mar;58(3):379-87.

29 Yang WH, Heithoff DM, Aziz PV, Sperandio M, Nizet V, Mahan MJ, et al. Recurrent infection progressively disables host protection against intestinal inflammation. Science. 2017 Dec;358(6370):eaao5610.

30 Ramasamy S, Nguyen DD, Eston MA, Alam SN, Moss AK, Ebrahimi F, et al. Intestinal alkaline phosphatase has beneficial effects in mouse models of chronic colitis. Inflamm Bowel Dis. 2011 Feb;17(2):532-42.

31 Alam SN, Yammine H, Moaven O, Ahmed R, Moss AK, Biswas B, et al. Intestinal alkaline phosphatase prevents antibiotic-induced susceptibility to enteric pathogens. Ann Surg. 2014 Apr;259(4):715-22.

32 Berman L, Moss RL. Necrotizing enterocolitis: an update. Semin Fetal Neonatal Med. 2011 Jun;16(3):145-50.

33 Biesterveld BE, Koehler SM, Heinzerling NP, Rentea RM, Fredrich K, Welak SR, et al. Intestinal alkaline phosphatase to treat necrotizing enterocolitis. J Surg Res. 2015 Jun;196(2): 235-40.

34 Rentea RM, Liedel JL, Fredrich K, Welak SR, Pritchard KA Jr, Oldham KT, et al. Intestinal alkaline phosphatase administration in newborns decreases systemic inflammatory cytokine expression in a neonatal necrotizing enterocolitis rat model. J Surg Res. 2012 Oct; 177(2):228-34.

35 Rentea RM, Liedel JL, Welak SR, Cassidy LD, Mayer AN, Pritchard KA Jr, et al. Intestinal alkaline phosphatase administration in newborns is protective of gut barrier function in a neonatal necrotizing enterocolitis rat model. J Pediatr Surg. 2012 Jun;47(6):1135-42.

36 Heath M, Buckley R, Gerber Z, Davis P, Linneman L, Gong Q, et al. Association of Intestinal Alkaline Phosphatase With Necrotizing Enterocolitis Among Premature Infants. JAMA Netw Open. 2019 Nov;2(11):e1914996.

37 Bilski J, Mazur-Bialy A, Wojcik D, Zahradnik-Bilska J, Brzozowski B, Magierowski M, et al. The Role of Intestinal Alkaline Phosphatase in Inflammatory Disorders of Gastrointestinal Tract. Mediators Inflamm. 2017; 2017:9074601.

38 Park SY, Kim JY, Lee SM, Chung JO, Seo JH, Kim S, et al. Lower expression of endogenous intestinal alkaline phosphatase may predict worse prognosis in patients with Crohn's disease. BMC Gastroenterol. 2018 Dec;18(1): 188.

39 Liu Y, Cavallaro PM, Kim BM, Liu T, Wang $\mathrm{H}$, Kühn F, et al. A role for intestinal alkaline phosphatase in preventing liver fibrosis. Theranostics. 2021 Jan;11(1):14-26.

40 Son G, Kremer M, Hines I N. Contribution of gut bacteria to liver pathobiology. Gastroenterol Res Pract. 2010;2010:453563.

41 Hamarneh SR, Kim BM, Kaliannan K, Morrison SA, Tantillo TJ, Tao Q, et al. Intestinal Alkaline Phosphatase Attenuates Alcohol-Induced Hepatosteatosis in Mice. Dig Dis Sci. 2017 Aug;62(8):2021-34.

42 Fukui H. Relation of endotoxin, endotoxin binding proteins and macrophages to severe alcoholic liver injury and multiple organ failure. Alcohol Clin Exp Res. 2005 Nov;29(11 Suppl):172S-9S

43 Sandahl TD, Grønbaek H, Møller HJ, Støy S, Thomsen KL, Dige AK, et al. Hepatic macrophage activation and the LPS pathway in patients with alcoholic hepatitis: a prospective cohort study. Am J Gastroenterol. 2014 Nov; 109(11):1749-56.

44 Nanji AA, Khettry U, Sadrzadeh SM, Yamanaka T. Severity of liver injury in experimental alcoholic liver disease. Correlation with plasma endotoxin, prostaglandin E2, leukotriene B4, and thromboxane B2. Am J Pathol. 1993 Feb;142(2):367-73.

45 Inokuchi S, Tsukamoto H, Park E, Liu ZX, Brenner DA, Seki E. Toll-like receptor 4 mediates alcohol-induced steatohepatitis through bone marrow-derived and endogenous liver cells in mice. Alcohol Clin Exp Res. 2011 Aug;35(8):1509-18.

46 Thurman RG. II. Alcoholic liver injury involves activation of Kupffer cells by endotoxin. Am J Physiol. 1998 Oct;275(4):G605-11.

47 Deaciuc IV, Nikolova-Karakashian M, Fortunato F, Lee EY, Hill DB, McClain CJ. Apopto- sis and dysregulated ceramide metabolism in a murine model of alcohol-enhanced lipopolysaccharide hepatotoxicity. Alcohol Clin Exp Res. 2000 Oct;24(10):1557-65.

48 Horie Y, Kato S, Ohki E, Tamai H, Ishii H. Role of endothelin in endotoxin-induced hepatic microvascular dysfunction in rats fed chronically with ethanol. J Gastroenterol Hepatol. 2001 Aug;16(8):916-22.

49 Horie Y, Kato S, Ohki E, Tamai H, Yamagishi Y, Ishii H. Hepatic microvascular dysfunction in endotoxemic rats after acute ethanol administration. Alcohol Clin Exp Res. 2000 May;24(5):691-8.

50 Aw W, Fukuda S. Understanding the role of the gut ecosystem in diabetes mellitus. J Diabetes Investig. 2018 Jan;9(1):5-12.

51 Capurso G, Signoretti M, Archibugi L, Stigliano S, Delle Fave G. Systematic review and meta-analysis: small intestinal bacterial overgrowth in chronic pancreatitis. United European Gastroenterol J. 2016 Oct;4(5): 697-705.

52 Kaliannan K, Hamarneh SR, Economopoulos KP, Nasrin Alam S, Moaven O, Patel P, et al. Intestinal alkaline phosphatase prevents metabolic syndrome in mice. Proc Natl Acad Sci USA. 2013 Apr;110(17):7003-8.

53 Malo MS. A High Level of Intestinal Alkaline Phosphatase Is Protective Against Type $2 \mathrm{Di}$ abetes Mellitus Irrespective of Obesity. EBioMedicine. 2015 Dec;2(12):2016-23.

54 Fawley J, Gourlay DM. Intestinal alkaline phosphatase: a summary of its role in clinical disease. J Surg Res. 2016 May;202(1):225-34.

55 Malo J, Alam MJ, Shahnaz M, Kaliannan K, Chandra G, Aziz T, et al. Intestinal Alkaline Phosphatase Deficiency Is Associated with Ischemic Heart Disease. Dis Markers. 2019 Dec;2019:8473565.

56 Economopoulos KP, Ward NL, Phillips CD, Teshager A, Patel P, Mohamed MM, et al. Prevention of antibiotic-associated metabolic syndrome in mice by intestinal alkaline phosphatase. Diabetes Obes Metab. 2016 May; 18(5):519-27.

57 Neu J, Reverte CM, Mackey AD, Liboni K, Tuhacek-Tenace LM, Hatch M, et al. Changes in intestinal morphology and permeability in the biobreeding rat before the onset of type 1 diabetes. J Pediatr Gastroenterol Nutr. 2005 May;40(5):589-95.

$58 \mathrm{Li} \mathrm{X}$, Atkinson MA. The role for gut permeability in the pathogenesis of type 1 diabetesa solid or leaky concept? Pediatr Diabetes. 2015 Nov;16(7):485-92.

59 Satoh M, Shimoda Y, Maesawa C, Akatsu T, Ishikawa Y, Minami Y, et al. Activated tolllike receptor 4 in monocytes is associated with heart failure after acute myocardial infarction. Int J Cardiol. 2006 May;109(2):226-34.

60 Fiechter D, Kats S, Brands R, van Middelaar B, Pasterkamp G, de Kleijn D, et al. Bovine Intestinal Alkaline Phosphatase Reduces Inflammation After Induction of Acute Myocardial Infarction in Mice. Cardiol Res. 2011 Oct;2(5):236-42.

61 Bentala H, Verweij WR, Huizinga-Van der Vlag A, van Loenen-Weemaes AM, Meijer DK, Poelstra K. Removal of phosphate from lipid A as a strategy to detoxify lipopolysaccharide. Shock. 2002 Dec;18(6):561-6. 
62 Kats S, Brands R, Hamad MA, Seinen W, Scharnhorst V, Wulkan RW, et al. Prophylactic treatment with alkaline phosphatase in cardiac surgery induces endogenous alkaline phosphatase release. Int J Artif Organs. 2012 Feb;35(2):144-51.

63 Presbitero A, Mancini E, Brands R, Krzhizhanovskaya VV, Sloot PM. Supplemented Alkaline Phosphatase Supports the Immune Response in Patients Undergoing Cardiac Surgery: Clinical and Computational Evidence. Front Immunol. 2018 Oct;9:2342.

64 Lehto M, Groop PH. The Gut-Kidney Axis: Putative Interconnections Between Gastrointestinal and Renal Disorders. Front Endocrinol (Lausanne). 2018 Sep;9:553.

65 Ghosh SS, Gehr TW, Ghosh S. Curcumin and chronic kidney disease (CKD): major mode of action through stimulating endogenous intestinal alkaline phosphatase. Molecules. 2014 Dec;19(12):20139-56.

66 Vallon V, Mühlbauer B, Osswald H. Adenosine and kidney function. Physiol Rev. 2006 Jul;86(3):901-40.

67 Peters E, Geraci S, Heemskerk S, Wilmer MJ, Bilos A, Kraenzlin B, et al. Alkaline phosphatase protects against renal inflammation through dephosphorylation of lipopolysaccharide and adenosine triphosphate. $\mathrm{Br} J$ Pharmacol. 2015 Oct;172(20):4932-45.
68 Peters E, Schirris T, van Asbeck AH, Gerretsen J, Eymael J, Ashikov A, et al. Effects of a human recombinant alkaline phosphatase during impaired mitochondrial function in human renal proximal tubule epithelial cells. Eur J Pharmacol. 2017 Feb;796:149-57.

69 Peters E, Ergin B, Kandil A, Gurel-Gurevin E, van Elsas A, Masereeuw R, et al. Effects of a human recombinant alkaline phosphatase on renal hemodynamics, oxygenation and inflammation in two models of acute kidney injury. Toxicol Appl Pharmacol. 2016 Dec;313: 88-96.

70 Villa CR, Ward WE, Comelli EM. Gut microbiota-bone axis. Crit Rev Food Sci Nutr. 2017 May;57(8):1664-72.

71 Brun LR, Brance ML, Rigalli A. Luminal calcium concentration controls intestinal calcium absorption by modification of intestinal alkaline phosphatase activity. Br J Nutr. 2012 Jul;108(2):229-33.

72 Brun LR, Lombarte M, Roma S, Perez F, Millán JL, Rigalli A. Increased calcium uptake and improved trabecular bone properties in intestinal alkaline phosphatase knockout mice. J Bone Miner Metab. 2018 Nov;36(6): 661-7.
73 Kuehn F, Adiliaghdam F, Hamarneh SR, Vasan R, Liu E, Liu Y, et al. Loss of Intestinal Alkaline Phosphatase Leads to Distinct Chronic Changes in Bone Phenotype. J Surg Res. 2018 Dec;232:325-31.

74 Larrick JW, Mendelsohn AR. Supplementation with Brush Border Enzyme Alkaline Phosphatase Slows Aging. Rejuvenation Res. 2020 Apr;23(2):171-5.

75 Adiliaghdam F, Cavallaro P, Mohad V, Almpani M, Kühn F, Gharedaghi MH, et al. Targeting the gut to prevent sepsis from a cutaneous burn. JCI Insight. 2020 Oct;5(19): 137128.

76 Ebrahimi F, Malo MS, Alam SN, Moss AK, Yammine H, Ramasamy S, et al. Local peritoneal irrigation with intestinal alkaline phosphatase is protective against peritonitis in mice. J Gastrointest Surg. 2011 May;15(5): 860-9.

77 Plaeke P, De Man JG, Smet A, Malhotra-Kumar S, Pintelon I, Timmermans JP, et al. Effects of intestinal alkaline phosphatase on intestinal barrier function in a cecal ligation and puncture (CLP)-induced mouse model for sepsis. Neurogastroenterol Motil. 2020 Mar; 32(3):e13754. 